\title{
ABSCESS FORMATION IN A PITUITARY ADENOMA
}

\author{
BY \\ NORMAN WHALLEY \\ From the Neurosurgical Unit, Swansea
}

The case described here is of particular interest for three reasons. First, suppuration within the sella turcica itself is an uncommon phenomenon, and suppuration within the substance of a pituitary adenoma must be extremely rare; I can find no reference to pyogenic abscess formation in a pituitary adenoma in an extensive search of the literature over the past 20 years. Secondly, in this case no complaint of deterioration of vision had been made until the onset of what appeared to be a fulminating type of meningitis, although the adenoma must have been present for a considerable time before the appearance of the acute illness. Blindness developed with great rapidity and was complete. Thirdly, the disease process resulted in a fulminating and rapidly fatal infection with little cellular reaction in the cerebrospinal fluid.

\section{Case History}

T.R.M., a man aged 50 years, had been in excellent health until two days before his admission to the Neurosurgical Unit, Newcastle-upon-Tyne. He had been at work until October 4, 1950, when he complained of headache and began to vomit, and his temperature rose rapidly to $103^{\circ} \mathrm{F}$. This was followed the next day by the rapid development of complete blindness in both eyes. At this time the clinical picture was that of meningitis : the neck was extremely stiff and Kernig's sign was strongly positive. In addition, both pupils were fixed and dilated. There was no papilloedema, but he had a left external rectus palsy. There was oedema of the left eyelid and a purulent post-nasal discharge. There is no record of the state of the deep tendon reflexes, but the superficial abdominal reflexes were depressed, and both plantar responses were extensor. Shortly after losing his vision he became unconscious, and at this point he was transferred to the Neurosurgical Unit on October $6,1950$.

On admission, the patient was semicomatose and clearly suffering from meningitis. The pulse rate was 80 per minute, and the temperature $105^{\circ} \mathrm{F}$. Both pupils were widely dilated and irresponsive to light stimulation directly and consensually. The left upper eyelid was swollen and oedematous, and appeared to be ptosed. It was impossible to test his ocular movements owing to his unconscious state. There was no papilloedema and no evidence of optic atrophy. All the deep tendon reflexes were depressed; the superficial abdominal reflexes were absent and the plantar responses were extensor in nature. Lumbar puncture showed a pressure of $120 \mathrm{~mm}$. cerebrospinal fluid; the cerebrospinal fluid was only slightly hazy and contained 154 cells per c.mm., $98 \%$ of the cells being polymorphonuclear leucocytes. The protein content of the fluid was 200 mg. per $100 \mathrm{ml}$. and there was a marked increase in globulin content. Shortly after admission the patient began to have twitchings of all four limbs which progressed into major epileptic attacks. He became deeply comatose, and died within 12 hours of admission. Radiographs of the skull were not made.

The clinical diagnosis was fulminating meningitis following cavernous sinus thrombosis presumably secondary to nasal sinus infection.

Necropsy.-A complete post-mortem examination wasmade by Dr. Paterson Smith, but only the findings within the cranium will be given since all other organs were normal.

There was a walnut-sized tumour occupying and protruding upwards from the sella turcica which was widely distended and its posterior wall thinned. The tumour measured $1.5 \mathrm{~cm}$. antero-posteriorly, $2 \mathrm{~cm}$. transversely, and protruded $1.5 \mathrm{~cm}$. above the sella turcica. It expanded at its base within the sella to $2 \mathrm{~cm}$. antero-posteriorly and $3 \mathrm{~cm}$. transversely. The vertical measurement, or whole depth of the tumour, was $3 \mathrm{~cm}$. of which $1.5 \mathrm{~cm}$. protruded outside the sella. It appeared to be well circumscribed.

The infundibulum of the pituitary gland appeared to be normal. The optic nerves were flattened and stretched over its surface which showed a purulent exudate. On the transverse (coronal) section the whole left side of the tumour was seen to contain a confluent abscess from which a swab was taken for culture (Fig. 1). The main mass of the tumour had a reddish homogeneous character resembling a true pituitary tumour rather than a craniopharyngioma. No small calcified areas could be seen and there were no cysts.

Portions of the abscess area and tumour were taken for section, and the specimen was photographed.

The tumour had not infiltrated bone or brain although it had formed a cavity at the base in the region of the 


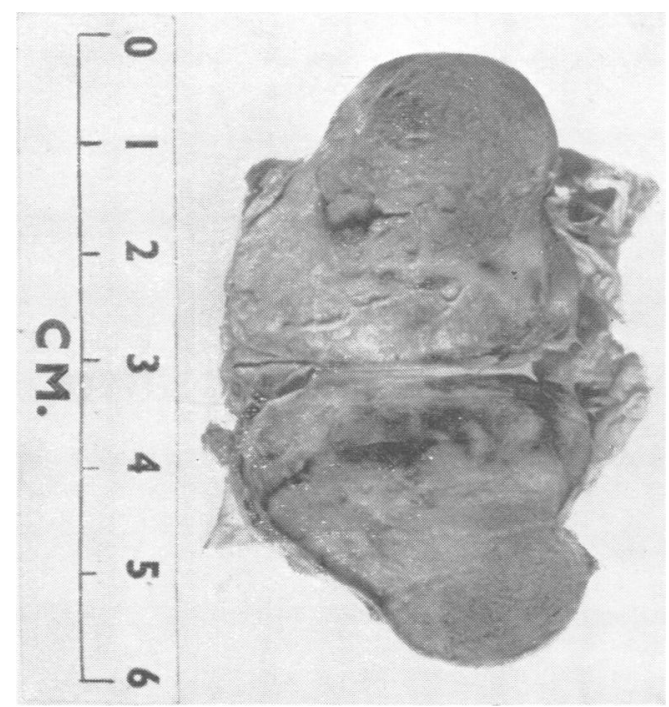

FIG. 1.-The adenoma has been removed and sectioned in the coronal plane to show the extension of the tumour upwards and slightly to the right, together with the confluent abscess on the left side. the pus at necropsy showed a growth of Bact. coli. No organisms were grown from the cerebrospinal fluid.

\section{Comment}

This patient had undoubtedly been harbouring a pituitary adenoma for some time although he had not complained of any interference with his vision. It is of course always possible that he had a small visual field defect which had passed unnoticed, but there was certainly no evidence of optic atrophy on ophthalmoscopy. In addition there were no clinical signs of pituitary dysfunction in the rest of the body. The formation of an abscess within the tumour no doubt caused the adenoma to grow rapidly due to the mass of suppuration and also to reactionary oedema around it, thus causing rapid compression and stretching of both optic nerves. The rapidity of his death and the signs of a fulminating infection can be accounted for by spread along the infundibulum to the third ventricle together with pressure on the hypothalamus.

This patient came under my care when I was deputy regional neurosurgeon at the Newcastle-upon-Tyne General Hospital, and I should like to thank Mr. G. F.

optic chiasma. Otherwise the brain was normal. There was no meningitis, hydrocephalus, or pressure cone.

Except for some mucopurulent material in the left sphenoidal air sinus, there was nothing else abnormal to be found in the skull nor was there any evidence of cavernous sinus thrombosis.

The pathological diagnosis was suprasellar and intrasellar chromophobe adenoma of the pituitary gland with abscess formation from extension of infection from the left sphenoidal air sinus which contained pus.

Histological Examination.-The tumour consisted of non-granular chromophobe cells, with a characteristic alveolar grouping and fibrous septa between the groups, though some of the cells were also arranged differently. There were also a few eosinophil and basophil cells, as well as areas of simple abscess formation

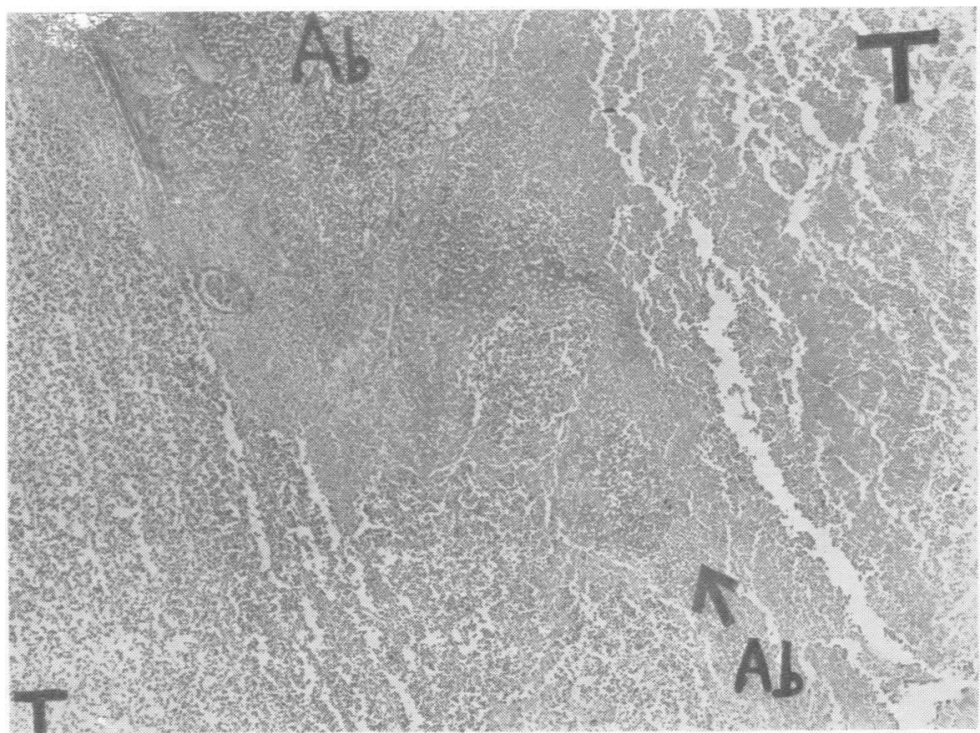

FiG. 2.-Chromophobe adenoma (T). Traversed by broad band of necrosis and inflammatory cells. ( $\times 34)$ containing many pus cells (Fig. 2).

The appearances were those of a simple chromophobe adenoma of the pituitary, showing abscess formation, apparently an extension from the left sphenoidal air sinus, which contained pus. There was no evidence of malignancy or tuberculosis. The culture obtained from

Rowbotham, the regional neurosurgeon, for his permission to publish the case record. In addition I should like to acknowledge my indebtedness to Dr. Paterson Smith, of the Newcastle-upon-Tyne General Hospital, for the pathological report. 